\title{
SYMPOSIUM
}

\section{Patterns of visual sensory and sensorimotor abnormalities in autism vary in relation to history of early language delay}

\author{
YUKARI TAKARAE, ${ }^{1}$ BEATRIZ LUNA, ${ }^{2,4}$ NANCY J. MINSHEW, ${ }^{2,3}$ AND JOHN A. SWEENEY 5,6,7,8 \\ ${ }^{1}$ Center for Mind and Brain, University of California, Davis, Davis, California \\ ${ }^{2}$ Department of Psychiatry, University of Pittsburgh, Pittsburgh, Pennsylvania \\ ${ }^{3}$ Department of Neurology, University of Pittsburgh, Pittsburgh, Pennsylvania \\ ${ }^{4}$ Department of Psychology, University of Pittsburgh, Pittsburgh, Pennsylvania \\ ${ }^{5}$ Center for Cognitive Medicine, University of Illinois at Chicago, Chicago, Illinois \\ ${ }^{6}$ Department of Psychiatry, University of Illinois at Chicago, Chicago, Illinois \\ ${ }^{7}$ Department of Neurology, University of Illinois at Chicago, Chicago, Illinois \\ ${ }^{8}$ Department of Psychology, University of Illinois at Chicago, Chicago, Illinois \\ (Received December 21, 2007; Final Revision July 7, 2008; Accepted July 8, 2008)
}

\begin{abstract}
Visual motion perception and pursuit eye movement deficits have been reported in autism. However, it is unclear whether these impairments are related to each other or to clinical symptoms of the disorder. High-functioning individuals with autism (41 with and 36 without delayed language acquisition) and 46 control subjects participated in the present study. All three subject groups were matched on chronological age and Full-Scale IQ. The autism group with delayed language acquisition had bilateral impairments on visual motion discrimination tasks, whereas the autism group without delay showed marginal impairments only in the left hemifield. Both autism groups showed difficulty tracking visual targets, but only the autism group without delayed language acquisition showed increased pursuit latencies and a failure to show the typical rightward directional advantage in pursuit. We observed correlations between performance on the visual perception and pursuit tasks in both autism groups. However, pursuit performance was correlated with manual motor skills only in the autism group with delayed language, suggesting that general sensorimotor or motor disturbances are a significant additional factor related to pursuit deficits in this subgroup. These findings suggest that there may be distinct neurocognitive phenotypes in autism associated with patterns of early language development. (JINS, 2008, 14, 980-989.)
\end{abstract}

Keywords: Autism phenotypes, Visual motion perception, Visual pursuit, Population heterogeneity, Language development, Autism subtypes

\section{INTRODUCTION}

Autism is a genetic disorder that is associated with impairments in sensorimotor behaviors as well as deficits in language, cognition and social skills. Impairments in postural control (Minshew et al., 2004; Molloy et al., 2003), eye movements (Goldberg et al., 2002; Luna et al., 2007; Takarae et al., 2004a), and motor learning (Mostofsky et al., 2000;

Correspondence and reprint requests to: Yukari Takarae, Ph.D., 267 Cousteau Place, Center for Mind and Brain, University of California, Davis, Davis, CA 95616. E-mail: ytakarae@ucdavis.edu
Müller et al., 2004; Rinehart et al., 2001); all have been reported in autism. Sensorimotor functions are supported by extensive cortical and subcortical networks. Because the neuroanatomy of sensorimotor systems are well understood, and sensory inputs and motor responses are more easily quantifiable than most higher cognitive processes, an examination of sensorimotor behaviors offers advantages to investigate what neural circuits are selectively affected in autism.

Whereas sensorimotor impairments have been reported in autism, it is not clear whether sensorimotor deficits in autism are primarily consequences of altered sensory input 
to sensorimotor systems, or result from intrinsic disturbances in sensorimotor systems themselves. For example, smooth pursuit eye movements, which maintain visual focus on moving objects, depend on the analysis of visual motion information and are initiated in response to visual movement (Ilg, 1997; Newsome et al., 1985). The emergence of smooth pursuit during development follows maturation of the extrastriate area MT that is dedicated to visual motion processing (Johnson, 1990), suggesting a closely coordinated timing in maturation in the abilities to perceive and respond to visual motion signals.

Several studies have reported impairments in visual pursuit in autism. Scharre \& Creedon (1992) and Rosenhall et al. (1988) reported difficulty inducing smooth pursuit in subjects with autism. Though the latter reported some normalization of pursuit accuracy once smooth pursuit was successfully induced, lack of pursuit responses in most subjects points to abnormality in the pursuit system. Kemner et al. (2004) studied smooth pursuit eye movements in 14 children with Pervasive Developmental Disorders (PDD) and 14 age and IQ matched control children. Whereas not statistically significant, they reported about a $10 \%$ reduction in pursuit gain (pursuit velocity relative to target velocity) in the PDD group. Takarae and colleagues (Takarae et al., 2004a) found pursuit impairments in 60 highfunctioning individuals with autism compared to 94 age and IQ matched typically developing control subjects. They reported unidirectional impairments during pursuit to the right during the initiation of smooth pursuit and bidirectional impairments in sustained pursuit driven by predictive signals.

Pursuit impairments in autism might be caused by disturbances in visual processing because past studies have reported impairments in visual motion perception in autism (Bertone et al., 2003; Milne et al., 2002; Spencer et al., 2000). The degree to which impairments in visual perception explain visual sensorimotor deficits in autism is, however, not clear. Our past work documented significant correlations between pursuit performance and manual motor skills in autism (Takarae et al., 2004a), suggesting that visual pursuit impairments might be part of more general visual sensorimotor or motor impairments. Milne et al. (Milne et al., 2006) reported abnormally high motion detection thresholds in only $22 \%$ of their participants, and whether or not individuals with more severe motion perception impairments have more severe pursuit impairments remains to be established. There is potential heterogeneity with respect to the development of sensorimotor and sensory brain systems in autism, in which each system could be more or less impaired to result in general sensorimotor disturbances.

The current study examined visual motion perception, visual pursuit, manual motor control, and relationships between these behavioral domains in autism. We used two different visual pursuit tasks because some aspects of visual pursuit are more sensitive to the quality of visual sensory input to the oculomotor system than others. Lesions in the area MT most profoundly affect latency of pursuit eye move- ments and pursuit gain during the initial $100 \mathrm{msec}$ from the onset of smooth pursuit (referred to as the open-loop stage) (Dursteler \& Wurtz, 1988; Newsome et al., 1985). Thus, we used two different paradigms to examine pursuit initiation and maintenance separately. We also used two different paradigms to examine visual motion processing, because previous literature has suggested that performance on visual motion tasks can depend on stimulus characteristics (Bertone et al., 2003). One task was a motion coherence task which requires integration of local motion vectors to compute global motion, and the other was based on detecting moving gratings which could be performed by detection of local motion alone. Because we previously found lateralized impairments during pursuit initiation, we assessed performance in the left and right hemifield separately during visual pursuit and visual motion tasks. To our knowledge, this is the first study that examined visual motion perception in each hemifield separately in autism. We also used neuropsychological measures of manual motor skills to replicate our previous findings of a relationship between manual motor skills and visual pursuit performance and to assess the generality of sensorimotor deficits across visuomotor systems.

Because of previous findings indicating that visual perception may be differentially affected in high-functioning autism and Asperger's Disorder (Spencer \& O'Brien, 2006), we divided participants meeting the Autism Diagnostic Interview-Revised (ADI-R) and the Autism Diagnostic Observation Schedule (ADOS) criteria for autism according to their history of early language development. That is one of critical factors differentiating the two clinical disorders. A diagnosis of Asperger's Disorder requires the absence of delayed language development, whereas DSM-IV criteria for Autistic Disorder do not require delayed language development. Thus, one could have a diagnosis of Autistic Disorder regardless of the presence or absence of delayed language development. In DSM-IV, diagnosis of Autistic Disorder supercedes Asperger's Disorder, and thus the difference between two disorders reflects both qualitative and quantitative differences. In the current study, we compared two autism groups with similar symptom severity on the ADOS rating scale.

Defining subgroups of individuals with autism based on language development has been proposed as a viable phenotype in autism by genetic and brain imaging studies as well as behavioral analysis (Tager-Flusberg \& Joseph, 2003), and those with language delay might have specific genetic alterations (Alarcon et al., 2002; Bradford et al., 2001). Our previous work on saccadic eye movements documented that autism groups that were defined by the presence or absence of early language delay were associated with a distinct pattern of neurophysiological impairments (Takarae et al., 2004b). Delay in language acquisition likely represents an observable clinical marker for changes in complex brain maturation at the age that early language skills are acquired; these have diverse implications for many neuropsychological processes. Effects involving language 
development and visual processing might occur via alteration in the superior temporal sulcus or its white matter connections that are implicated in autism and are associated with both verbal abilities and high-level visual processing (Redcay, 2008; Zilbovicius et al., 2006). In the present study, we examined whether problems in early language acquisition are related to impairments in visual pursuit and visual motion perception.

\section{METHODS}

\section{Participants}

Participants were 77 individuals with autism and 46 typically developing individuals matched for age and FullScale IQ from age-appropriate versions of the Wechsler Intelligence Scale (Table 1). One control subject and 4 subjects with autism that were included in our previous work on smooth pursuit eye movements (Takarae et al., 2004a) were retested for this study, and the remaining 118 subjects were newly recruited. The diagnosis of autism was established by expert clinical opinion verified by results from the ADI-R and ADOS (Lord et al., 1989; Lord et al., 1994; Lord et al., 2000). Subjects were excluded if they had an associated infectious, genetic, or metabolic disorder known to cause autistic features such as fragile $\mathrm{X}$ syndrome or tuberous sclerosis. Individuals with autism were divided into two groups depending on the presence of delay in language acquisition in their developmental history. Language delay was defined as the absence of single word utterances by 24 months and/or spoken phrases by three years of age. This resulted in a group of 41 individuals with delay in language acquisition and 36 individuals without delay. Average ADOS scores for these groups were similar (Table 1).

Typically developing control participants had no per- sonal history of psychiatric or neurological disorder, no family history of autism, and no first-degree relatives with any neuropsychiatric disorder considered to have a genetic component. They had no personal history of developmental delay, significant problems in school performance, or signs of learning disability in psychoeducational testing that included language tests as described in our previous reports (Minshew et al., 1997; Williams et al., 2006). No participants were taking stimulant, lithium, antipsychotic, or anticonvulsant medications at the time of testing. Twelve subjects with autism were taking antidepressants. No participant had a history of head injury, birth injury, or seizure disorder. Far acuity of all participants was normal or corrected to at least 20/40. After providing the complete description of the study, informed consent (or assent) was obtained from all participants and consent was obtained for parents/guardians for those under 18 years of age. The study was approved by the Institutional Review Boards of the University of Pittsburgh and University of Illinois at Chicago.

\section{Procedures}

Participants were tested in a darkened flat black room, with task instructions provided via intercom. Targets for pursuit paradigms were presented in the horizontal plane at eye level on an acrylic hemi-arc with 1-meter radius. Participants were seated at the center of the arc. A laser diode module was mounted immediately over participants' heads to produce a 3-mm point source of light. The light source was reflected onto the arc by the use of a mirror attached to a rotary stage assembly (New England Affiliated Technologies \#1121-142, Lawrence, MA) that manipulated velocity profiles and locations of targets. Stimuli for visual motion tasks were presented using a CRT monitor (SONY, GDMFW900 Multiscan, New York, NY) with subjects seated 27 " distance from the monitor.

Table 1. Demographic and neuropsychological characteristics of participants

\begin{tabular}{|c|c|c|c|c|}
\hline & Control & $\begin{array}{l}\text { Autism with } \\
\text { language delay }\end{array}$ & $\begin{array}{l}\text { Autism without } \\
\text { language delay }\end{array}$ & Statistics \\
\hline Age & $16.54(5.98)$ & $16.30(7.18)$ & $15.25(5.42)$ & $F<1$, n.s. \\
\hline Full-Scale IQ & $107.41(7.97)$ & $101.78(12.78)$ & $103.58(13.20)$ & $F(2,120)=2.80$, n.s. \\
\hline ADOS Communication & $\mathrm{N} / \mathrm{A}$ & $4.66(1.06)$ & $4.58(1.16)$ & $t<1$, n.s. \\
\hline ADOS Social & $\mathrm{N} / \mathrm{A}$ & $9.83(1.83)$ & $9.39(2.05)$ & $t<1$, n.s. \\
\hline ADOS Comm + Social & $\mathrm{N} / \mathrm{A}$ & $14.49(2.49)$ & $13.97(2.49)$ & $t<1$, n.s. \\
\hline$\%$ Male & $84.8 \%$ & $90.2 \%$ & $86.1 \%$ & $\chi^{2}(2)=.606$, n.s. \\
\hline $\begin{array}{l}\text { Finger tapping dominant hand } \\
\quad(\text { taps per } 10 \mathrm{sec})\end{array}$ & $46.16(10.53)$ & $45.33(9.88)$ & $44.91(8.96)$ & $\begin{array}{l}\text { F's }<1 \text { for Group, } \\
\text { Group } \times \text { Hand }\end{array}$ \\
\hline $\begin{array}{l}\text { Finger tapping non-dominant hand } \\
\quad(\text { taps per } 10 \mathrm{sec})\end{array}$ & $43.19(10.24)$ & $41.64(8.51)$ & $42.60(8.13)$ & \\
\hline $\begin{array}{l}\text { Grooved Pegboard Dominant Hand } \\
\text { (time to complete in secs) }\end{array}$ & $70.81(10.66)$ & $93.10(27.10)$ & $87.91(22.26)$ & $\begin{array}{l}F(2,92)=11.40, \\
p<.001 \text { for Group, } \\
F<1 \text { for Group } \times \text { Hand }\end{array}$ \\
\hline $\begin{array}{l}\text { Grooved Pegboard Non-Dominant Hand } \\
\text { (time to complete in secs) }\end{array}$ & $79.26(13.42)$ & $101.97(29.47)$ & $101.50(37.58)$ & \\
\hline
\end{tabular}


Eye movements were monitored using infrared reflection sensors mounted on spectacle frames (Applied Science Laboratories, Inc., Model 210, Bedford, MA) during eye movement and visual motion tasks. Subjects' heads were secured in a chinrest with a head strap to minimize head movement. An experimenter monitored eye movement activity throughout testing to ensure that participants were alert and performing tasks according to instructions. Eye movement recordings were digitized at $500 \mathrm{~Hz}$ with a 16 bit A/D converter (Dataq Instruments, DI-720, Akron, OH) and processed with a custom finite impulse response filter. Details of the data processing stream and algorithm used to classify eye movements are presented elsewhere (Takarae et al., 2004a).

\section{Eye Movement Tasks}

\section{Foveofugal Step-ramp Pursuit Tracking Task}

Targets were presented at center for $2-4$ seconds, and then stepped 3 degrees to the left or right and immediately continued moving in the same direction away from center at a constant speed of $4,8,16$, or $24 \%$ sec (Fig. 1). The target disappeared after reaching $\pm 15^{\circ}$. One second later, the target reappeared at center to begin the next trial. The duration of central fixation, and the speed and direction of target movement, were pseudorandomly assigned for each trial.

In this paradigm, a saccade typically occurs approximately $200 \mathrm{msec}$ after the onset of target motion so that the eyes can catch up to the moving target, followed immediately by smooth pursuit. The initial phase of the pursuit

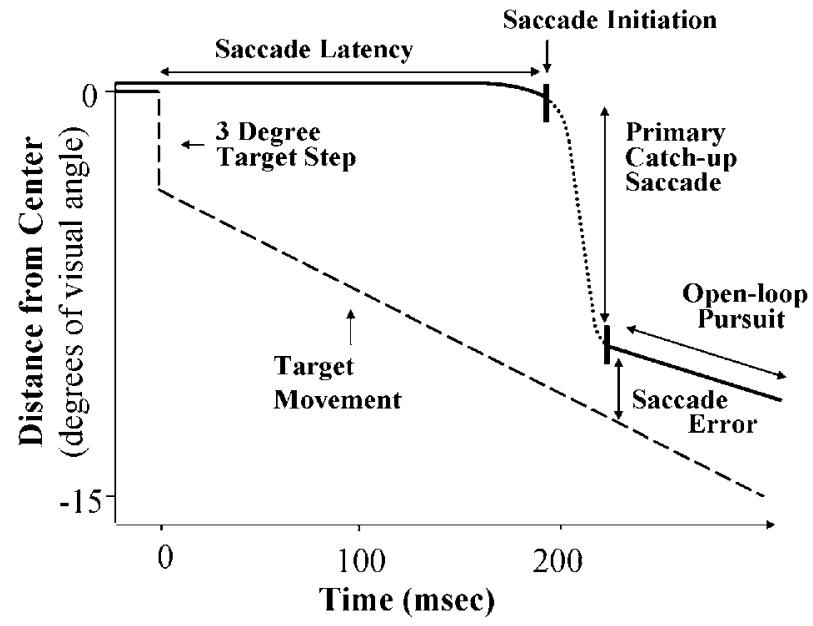

Fig. 1. Schematic presentation of a foveofugal step ramp task. The diagram shows a time (horizontal axis) by target position (vertical axis) plot for a single trial of this task. The dashed line represents target movement with an initial $3^{\circ}$ step followed by smooth movement towards the left (negative) 15-degree position. The line above illustrates a typical eye movement response in which an initial catch-up saccade (dotted line) to the target is followed by smooth pursuit. response (defined here as the first $100 \mathrm{msec}$ of smooth pursuit after the end of the first catch-up saccade) is referred to as the open-loop stage because it is primarily driven by sensory input. Pursuit after that point, the closed-loop period, is driven primarily by internally generated feedback about performance accuracy and predictions about target motion. This task has been extensively used in studies of visual motion perception with non-human primates, and openloop pursuit becomes severely impaired after lesions in extrastriate area MT (Newsome et al., 1985). In contrast, damage to premotor areas, such as the frontal eye fields, tend to produce deficits that are consistent in the open- and closedloop stages of visual pursuit (Lynch, 1987; Rivaud et al., 1994). Primary measures of performance were pursuit gain from the open-loop and closed-loop stages, which was defined as the ratio of average pursuit eye movement velocity over target velocity. This task consisted of a total of 32 trials (4 trials $\times 4$ velocities $\times 2$ directions).

\section{Pure Ramp Task}

Targets were presented at the central location for 2-4 seconds, and then swept to the left or right at a constant speed $(4,8,16,24$, or $32 \% \mathrm{sec})$ and then terminated after reaching $\pm 15^{\circ}$ (Fig. 2). This task was designed to measure visually guided pursuit initiation, a parameter whose latency is prolonged by lesions to visual cortex (Heide et al., 1996; Lisberger \& Pavelko, 1989). Thus, pursuit latency was used as a primary measure for the reactivity of visual pursuit systems to visual motion and was defined as the time for the eyes to achieve a pursuit velocity of at least 2 degrees/sec

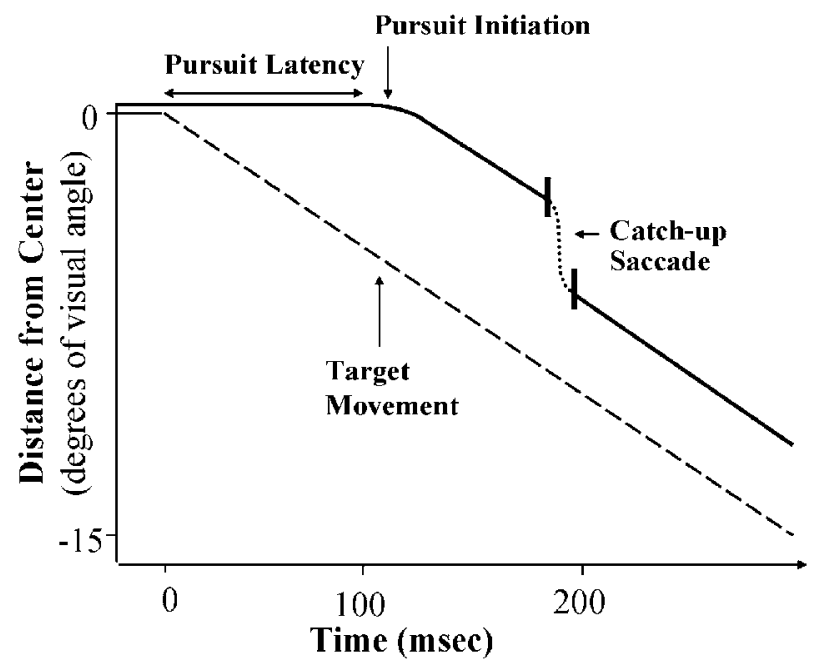

Fig. 2. Schematic presentation of a pure ramp task. The diagram shows a time (horizontal axis) by target position (vertical axis) plot for a trial with a target moving from center to a left (negative) 15-degree position. The dashed line shows target movement. The line above shows a trial where smooth pursuit is sometimes interrupted by a catch-up saccade that corrects for tracking error (shown in a dotted line). 
that was maintained for at least $20 \mathrm{msec}$. Average pursuit gain and the number of catch-up saccades made to compensate for slow pursuit were also obtained. Forty trials were performed (4 trials $\times 5$ velocities $\times 2$ directions). In both visual pursuit tasks, each trial began with presentation of a $0.5 \mathrm{sec}$ auditory tone at center fixation concurrently with the presentation of the central visual target to alert participants to prepare for the next trial.

\section{Visual Motion Perception Tasks}

Of 123 subjects who completed the pursuit tasks, 110 subjects (46 controls, 37 subjects with autism who had language delay, and 27 subjects with autism who did not have language delay) completed the motion perception tasks. ANOVAs on age and Full-Scale IQ scores revealed no statistical difference across groups $(F$ 's $<1)$.

\section{Dynamic Contrast Task}

Participants were presented with gratings in two wedgeshaped windows $\left(0.67\right.$ cycles per $\mathrm{deg}, 5^{\circ}$ from center in the widest extent) on the left and right sides of the central fixation cross $\left(0.85^{\circ}\right.$ wide; see Fig. 3a for illustration). During

\section{a) Dynamic Contrast Task}

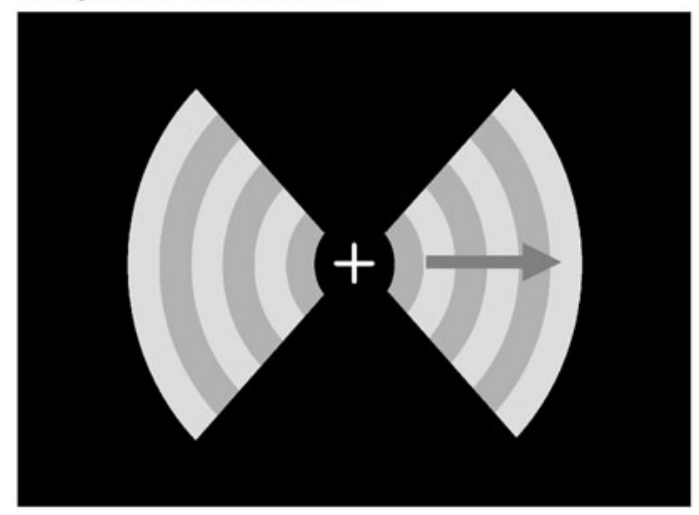

b) Motion Coherence Task

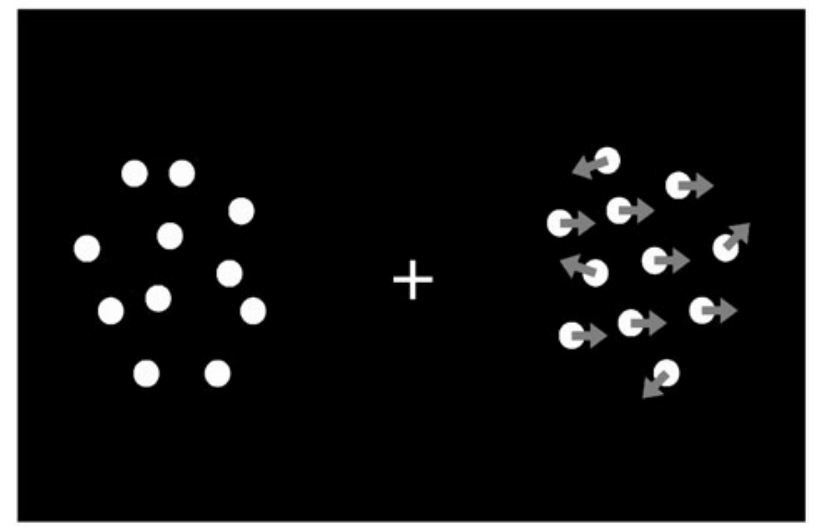

Fig. 3. Schematic presentations of a) the Dynamic Contrast Task and b) the Motion Coherence Task used in the current study. Both figures illustrate the right hemifield trial with rightward movement. a trial, the grating in the left or right hemifield moved at $1 \%$ towards the left or right directions for $300 \mathrm{~ms}$. On termination of the movement, participants were instructed to press a button to indicate the direction of movement regardless of the hemifield in which movement was presented. Visual fields in which movement occurred and direction of movement were pseudorandomly and randomly assigned respectively to each trial. Target movement was presented only after participants maintained their eye position within two degrees from center fixation for at least 1 sec. Target movement was terminated immediately if the participant moved their eyes from center fixation during the trial. The task resumed after the participant re-fixated on the center target. This was done to ensure that visual stimuli were presented in a similar location in the hemifield of interest across trials. The starting contrast level was $10 \%$. Contrast levels of the gratings were reduced using the two down one up staircase procedure, which yields thresholds to achieve $71 \%$ accuracy (Levitt, 1971). Contrast levels were manipulated using 30\% increments for the first 4 reversals then reduced to $15 \%$ increments thereafter until eight more reversals were observed. The staircase procedure was applied separately to left and right visual field trials to calculate discrimination thresholds for each hemifield separately. The average of the last six reversals for each staircase was used as an index of the perceptual threshold for visual motion discrimination.

\section{Motion Coherence Task}

Stimuli for the motion coherence task were presented in an identical manner except for the following. Participants were presented with a group of 100 randomly placed dots $(0.15$ degrees in diameter) in high contrast inside invisible circles (7.5 degrees diameter) located in each hemifield (Fig. 3b). In each trial, left or rightward movement occurred for 300 $\mathrm{msec}$ in one of the hemifields at $3.3 \%$. Each dot was displayed for $100 \mathrm{~ms}$ and was replaced with a new dot generated at a random location after the $100 \mathrm{~ms}$ period in order to prevent subjects from tracking specific dots. The task started at the 100 coherence level where all dots were moving in the same direction. The number of dots moving in the same direction was reduced using the staircase procedure described above to estimate the discrimination threshold.

\section{Manual Motor Tasks}

\section{Finger Tapping Task}

Participants placed their index finger on a key and were asked to tap the key as fast as they could. This task from the Halstead-Reitan battery was performed with the dominant and non-dominant hand in blocks of $10 \mathrm{~s}$. A total of five blocks were performed with each hand, and the average number of taps per $10 \mathrm{~s}$ was used as a dependent measure. 


\section{Grooved Pegboard Task}

Participants were presented with a $5 \times 5$ set of slots that had various orientations and were asked to insert a peg to each slot. Each peg had a ridge on one side, which required participants to rotate the peg to fit the slot. The task was performed with the dominant and non-dominant hand, and the time to complete the task with each hand was measured.

\section{RESULTS}

\section{Eye Movement Tasks}

\section{Foveofugal Step-Ramp Pursuit Tracking Task}

There was a significant Speed $\times$ Group interaction in openloop pursuit gain, $F(6,366)=2.13, p<.05$, which stemmed from lower gain at slower target speeds in the autism group with language delay compared to the autism group without language delay (Fig. 4). Neither group with autism was statistically different from the control group. No other statistical difference was identified.

During closed-loop pursuit on this task, we identified a significant Direction $\times$ Group interaction in pursuit gain, $F(2,117)=4.24, p<.05$ (Fig. 5). Both the control group and autism group with language delay showed rightward advantage in performance while the autism group without language delay showed more symmetric performance. When the average gain from leftward and rightward trials were compared, the lateral difference was significant in the control group $(t(45)=3.77, p<.001)$ and autism group with language delay $(t(40)=3.57, p=.001)$, but not in the autism group without language delay $(t<1)$. The autism group without language delay had significantly lower gain

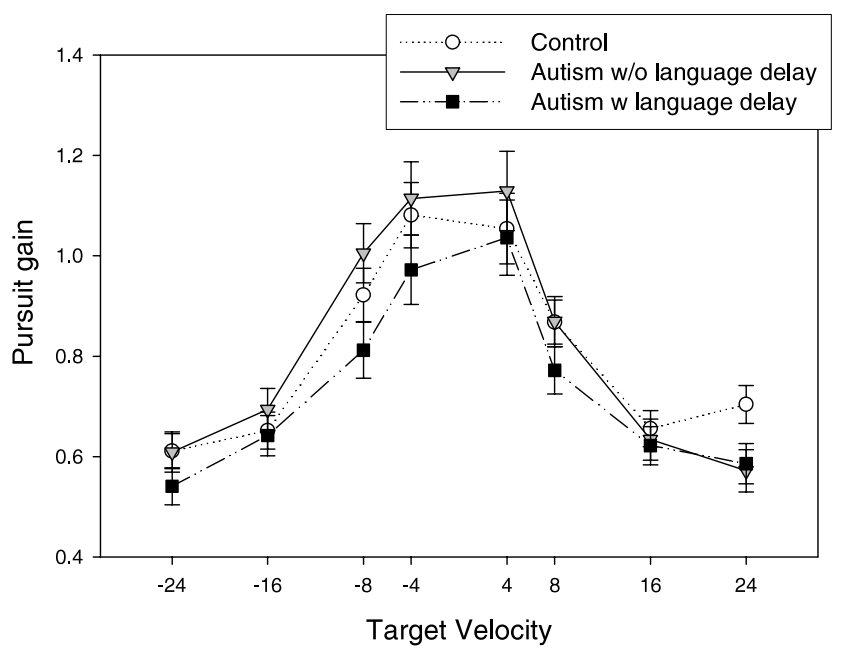

Fig. 4. Pursuit gain during the open-loop stage from the foveofugal step ramp task. Target velocities toward the right are shown in positive numbers and those toward the left are shown in negative numbers along the $\mathrm{X}$-axis. Pursuit gain was defined as a ratio of eye velocity over target velocity. For instance, pursuit gain of. 8 indicates that eye velocity was $20 \%$ lower than the target velocity.

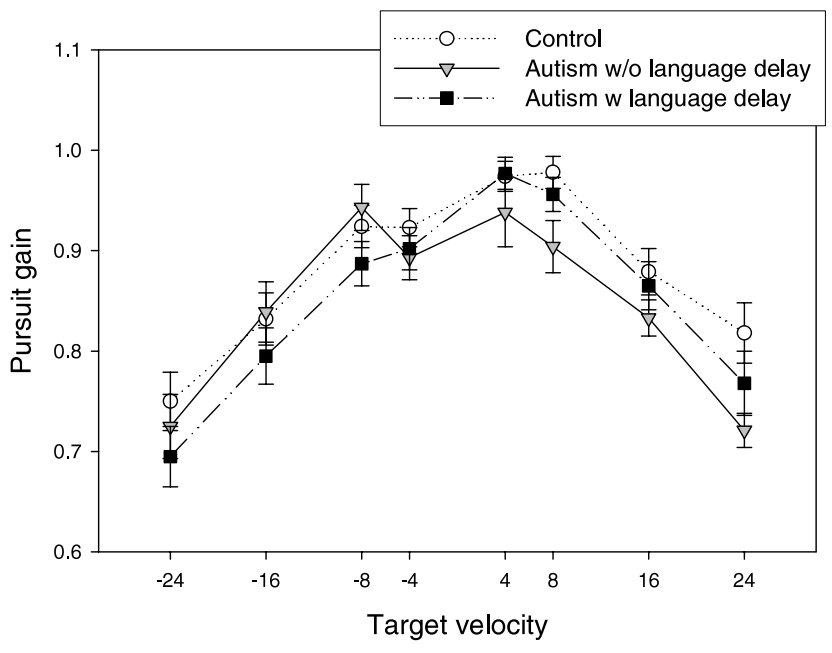

Fig. 5. Pursuit gain during the closed-loop stage from the foveofugal step ramp task.

in rightward trials than the control group, $t(118)=2.42$, $p<.05$. Whereas we did not find differences between the control group and either autism groups during open-loop pursuit, which is sensitive to impairments in visual motion perception, we observed an absence of the typical left/right asymmetry in closed-loop pursuit in the autism group without language delay.

\section{Pure Ramp Task}

There was a trend for the Group $\times$ Speed $\times$ Direction interaction in pursuit latency (Fig. 6), which is sensitive to disturbances in visual motion perception systems, with the autism group without language delay having longer latency than other groups in most target velocity conditions, $F(8,308)$ $=1.94, p=.053$. There was also a trend for group differences in average pursuit gain, $F(2,118)=2.87, p=.061$, with the autism group without language delay showing poorer performance than the control group (posthoc linear contrast, $p<.05$ ) (Fig. 7). There were also differences in the number of catch-up saccades that subjects made in order to keep up with the target, $F(2,119)=3.28, p<.05$. Both autism groups made higher numbers of catch-up saccades than the control group (linear contrasts, $p$ 's $<.05$ ). Though pursuit gain was only marginally lower than the control group, the significantly higher number of catch-up saccades made during this task suggests that both autism groups had difficulty tracking visual targets.

\section{Visual Motion Perception Tasks}

Visual thresholds obtained from the motion tasks were transformed by taking reciprocals to correct for non-linearity of the measures and skewness of the distributions, which is commonly performed in psychophysical studies (e.g., Chen et al., 1999). After the transformation, Group $\times$ Hemifield ANOVAs were used to compare performance across groups and hemifields. 


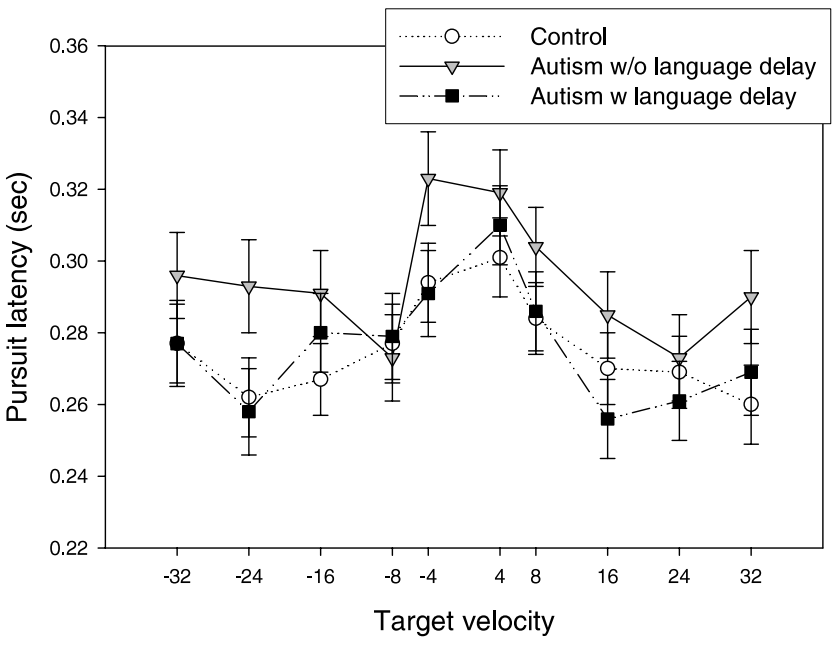

Fig. 6. Pursuit latency during the pure ramp task.

Group differences were detected when the threshold to discriminate directions of visual movement was assessed by varying the contrast of gratings on the Dynamic Contrast task, $F(2,92)=6.26, p<.01$ (Fig. 8). Posthoc linear contrasts showed that the autism group with language delay had poorer performance than the control group $(p=.001)$. There was no significant difference between the control group and the autism group without language delay. There was a trend for a Group $\times$ Hemifield interaction, $F(2,92)=$ $2.59, p=.081$. The marginal interaction was caused by a somewhat more asymmetric performance in the autism group without language delay, with poorer performance in the left than right hemifield $(t(21)=2.40, p=.078)$ while the other two groups showed similar performances across hemifields.

When the threshold to discriminate directions of visual motion was measured by the motion coherence task, there was a trend for a group difference in performance, $F(2,95)=$

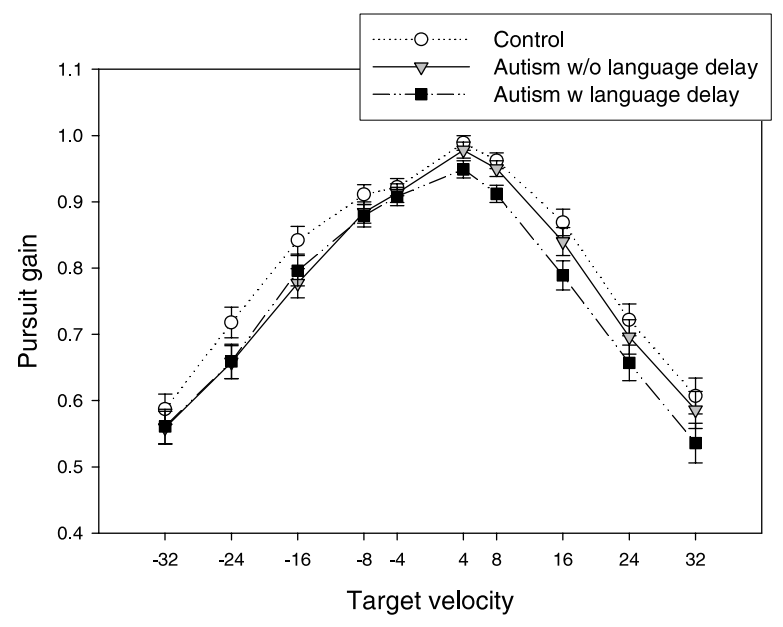

Fig. 7. Average pursuit gain during the pure ramp task.

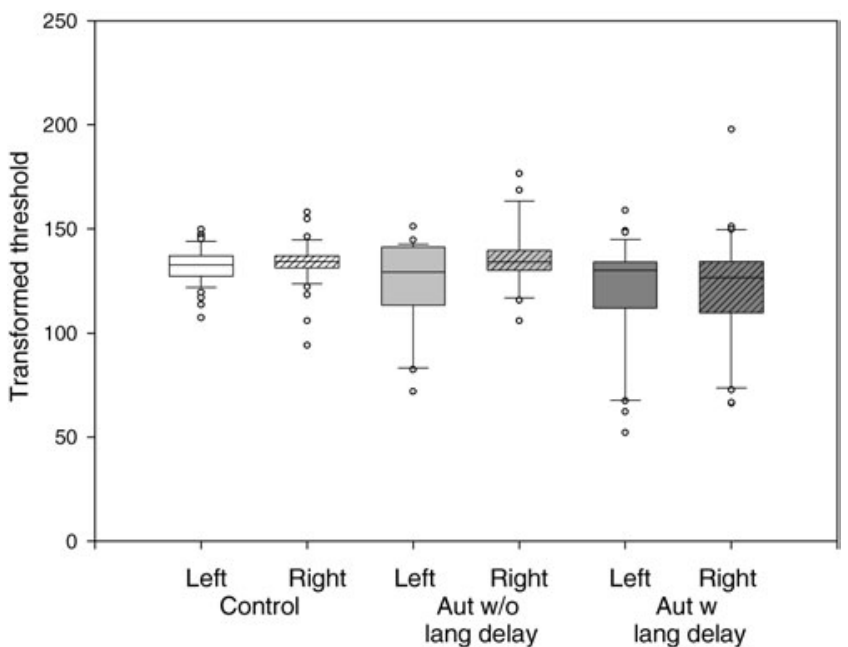

Fig. 8. Performance on the Dynamic Contrast task. Transformed thresholds for left and right hemifield conditions are shown separately. Higher transformed thresholds represent superior sensitivity to discriminate visual motion.

2.58, $p=.081$ (Fig. 9). The autism group with language delay had poorer performance than the control group $(p<$ $.05)$ whereas there was no difference between the control group and the autism group without language delay. No other statistical differences were found.

\section{Correlational Analyses}

In order to investigate possible relationships between impairments in visual pursuit and visual motion perception, we performed exploratory correlational analyses using a

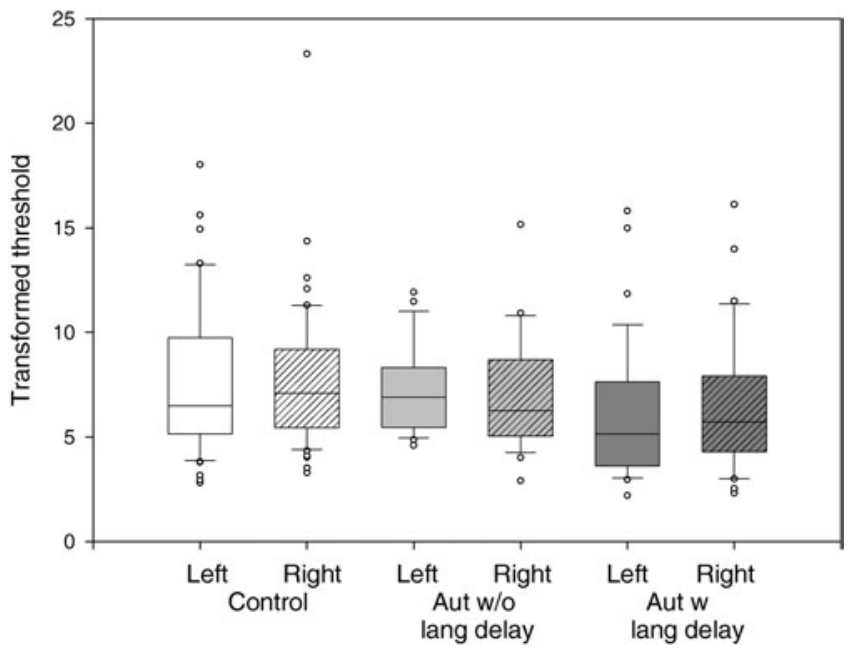

Fig. 9. Performance on the Motion Coherence task. Transformed thresholds for left and right hemifield conditions are shown separately. Higher transformed thresholds represent superior sensitivity to discriminate visual motion. 
$p<.05$ threshold. Because the initial open-loop pursuit performance has the highest reliance on sensory information, we used open-loop gain from the foveofugal step ramp task (Fig. 4) and pursuit latency from the pure ramp task (Fig. 6) in these analyses. Significant negative correlations were observed between performance on the Dynamic Contrast task in the left hemifield and pursuit latency in leftward trials (i.e., lower sensitivity to visual motion predicted longer pursuit latency), in the autism group with language delay $r(30)=-.44, p<.05$ and in the autism group without language delay, $r(22)=-.55, p<.01$. This effect was not significant in typically developing individuals, and no other correlations were significant.

Correlations between the pursuit measures and manual motor skills were also computed because our previous work with an independent data set (Takarae et al., 2004a) demonstrated a significant intercorrelation between oculomotor and manual motor measures in autism. Means and standard deviations of the manual motor measures are reported in Table 1. Both autism groups were slower to complete the Grooved Pegboard task than the control group $(F(2,92)=$ $11.40, p<.001$ ) whereas the number of taps per 10 seconds from the Finger Tapping task was similar between groups. There was no significant Group $\times$ Hand interaction on these tasks.

In the correlational analyses, no significant correlations were observed in the control group. In the autism group with language delay, time to complete the Grooved Pegboard task with the dominant hand was correlated with pursuit latency from the pure ramp task in rightward $(r(30)=$ $.40, p<.05)$ and leftward trials, $r(30)=.48, p<.01$. The time to complete measures with the non-dominant hand was also correlated with pursuit latency in rightward $(r(30)=$ $.40, p<.05)$ and leftward trials, $r(30)=.39, p<.05$. Additionally, performance on the Finger Tapping task with the non-dominant hand was correlated with open-loop pursuit gain from the foveofugal step ramp task in leftward trials, $r(33)=.40, p<.05$. In the autism group without language delay, there were no significant correlations between eye movement measures and manual neuropsychological test performance.

\section{DISCUSSION}

We examined visual motion perception, visual pursuit, and manual motor skills in high-functioning individuals who met ADOS and ADI criteria for autism. We observed pursuit impairments in both groups with autism though the pattern of impairments was somewhat different between them. Both autism groups had a general problem tracking moving targets as reflected in increased numbers of catch-up saccades and marginally reduced pursuit gain during the pure ramp task. The two autism groups, however, differed in two aspects. First, the autism group without language delay took marginally longer to initiate pursuit eye movement in response to visual motion in the pure ramp task. Increased pursuit latency, which is one of the primary chronic deficits observed after MT lesions (Heide et al., 1996; Lisberger \& Pavelko, 1989), suggests that at least some aspects of pursuit impairments in this group might be related to altered sensory input. Second, this group showed abnormal asymmetry in closed-loop gain from the step ramp task in which they lacked the typical rightward bias seen in controls and the group with language delay. Reduced pursuit gain in rightward trials in the autism group without language delay might point to a unique alteration in the development of normal hemispheric specialization or a right hemisphere dysfunction as the pursuit system has an ipsiversive organization. In contrast, the group with language delay did not have increased pursuit latency or lateralized impairments in pursuit gain though they did have general problems in visual pursuit that were correlated with manual motor skills.

Previous studies have shown some inconsistency with regards to reports of pursuit deficits in autism. Scharre and Creedon (1992) and Rosenhall et al. (1988) documented difficulty in eliciting pursuit responses in the majority of subjects (29 out of 34 in Scharre and Creedon and 7 out of 11 children in Rosenhall et al.) whereas a more recent study by Kemner et al. (2004) reported no statistical difference in pursuit accuracy in their subjects with PDD. Findings from these studies were inconclusive because of use of small samples or single measures to characterize subjects' performance. A large sample study by Takarae et al. (2004a) (60 subjects with autism and 94 control subjects) used three different pursuit tasks to characterize multiple aspects of pursuit performance. They have found reduced pursuit gain in three different tasks during the closed-loop stage where pursuit performance is mostly supported by non-sensory factors, primarily predictions about target motion. They also found reduced pursuit gain during the open-loop stage where the performance is primarily driven by sensory information that was specific to trials in which subjects tracked targets to the right. The current study with another largely independent sample also found pursuit impairments in autism. Whereas patterns of impairments are somewhat different across studies, possibly related to variability within the autism population, the two large sample studies suggest that pursuit eye movement impairments are neurophysiological characteristics of autism.

The present study also examined motion perception in autism and identified impairments in this behavioral domain. Although in contrast to pursuit studies where alterations in pursuit initiation and lateral asymmetry were largely restricted to the group without early language delay, motion processing deficits were relatively specific to the group with early language delay. The autism group without a history of early language delay had much milder impairments, with statistically marginal reduction in motion sensitivity only in the left hemifield during the Dynamic Contrast task. Unlike some previous studies (Milne et al., 2002; Spencer et al., 2000), our study did not find deficits in motion perception using a motion coherence task. This 
pattern of findings is inconsistent with the view that there is a selective impairment in higher level motion processing in autism, whereas lower level processing is spared (Bertone et al., 2003). Past findings that used motion coherence tasks have been inconsistently documenting impairments in autism (de Jonge et al., 2007; Milne et al., 2006), suggesting heterogeneity in sensitivity to visual motion in autism. Some studies have suggested that impairments in visual motion perception are limited to those with autism, and not found in Asperger's Disorder (Spencer \& O'Brien, 2006; Tsermentseli et al., in press). Similar to these findings, though all our subjects met criteria for Autistic Disorder based on DSM-IV criteria, we found more severe impairments in visual motion perception in the autism group with language delay.

These observations, along with different patterns of performance during visual pursuit tasks, suggest that early language delay might be associated with different patterns of brain development in autism spectrum disorders. Our correlational analyses showed relationships between pursuit performance and manual motor skills only in the autism group with language delay, suggesting a more generalized pattern of sensorimotor deficits in this subgroup of participants with autism. In contrast to this more generalized pattern of sensorimotor deficit, the autism group without language delay had abnormal asymmetry in some aspects of visual motion perception and visual pursuit, which point to disturbances in right hemisphere. The inference of selective right hemisphere dysfunction in this subgroup is based on the observed impairments in rightward pursuit and marginal impairments in motion perception in the left hemifield, as sensorimotor control of smooth pursuit is ipsiversively organized while visual sensory systems have contralateral organization (Ilg \& Schumann, 2007; Shi et al., 1998).

Observations from the current study provide evidence for a neurophysiological differentiation between individuals with autism in their associated visual sensory and sensorimotor impairments based on their history of early language delay. Those with language delay had more severe problems in visual motion perception, and their pursuit impairments were associated with more generalized disturbances in motor or sensorimotor systems as well as sensory processing disturbances. Those without a history of language delay showed abnormal asymmetries in performance during some aspects of pursuit and visual motion perception consistent with right hemisphere dysfunction and did not show correlations between pursuit and manual motor performance. Thus, brain dysmaturation in autism with language delay may be associated with more general alteration of brain development including premotor systems in frontal cortex, while autism without language delay may be associated with alteration of development of more posterior cortical areas, possibly more pronounced in the right hemisphere. The potential clinical relevance and genetic implications for this differentiation remain to be explored in future studies.

\section{ACKNOWLEDGMENTS}

Information that is presented in this manuscript and the manuscript itself has not been published elsewhere either electronically or in print. Authors do not have any financial or other conflicts that could affect this manuscript. This research was funded by an NICHD Collaborative Program of Excellence in Autism (HD35469) and grants MH01433 and NS33355 from the National Institute of Health, and the National Alliance for Autism Research (now Autism Speaks).

\section{REFERENCES}

Alarcon, M., Cantor, R.M., Liu, J., Gilliam, T.C., \& Geschwind, D.H. (2002). Evidence for a language quantitative trait locus on chromosome 7q in multiplex autism families. American Journal of Human Geneics, 70, 60-71.

Bertone, A., Mottron, L., Jelenic, P., \& Faubert, J. (2003). Motion perception in autism: A "complex" issue. Journal of Cognitive Neuroscience, 15, 218-225.

Bradford, Y., Haines, J., Hutcheson, H., Gardiner, M., Braun, T., Sheffield, V., Cassavant, T., Huang, W., Wang, K., Vieland, V., Folstein, S., Santangelo, S., \& Piven, J. (2001). Incorporating language phenotypes strengthens evidence of linkage to autism. American Journal of Medical Genetics (Neuropsychiatric Genetics), 105, 539-547.

Chen, Y., Palafox, G.P., Nakayama, K., Levy, D.L., Matthysse, S., \& Holzman, P.S. (1999). Motion perception in schizophrenia. Archives of General Psychiatry, 56, 149-154.

de Jonge, M.V., Kemner, C., de Haan, E.H., Coppens, J.E., van den Berg, T.J., \& van England, H. (2007). Visual information processing in high-functioning individuals with autism spectrum disorders and their parents. Neuropsychology, 21, 65-73.

Dursteler, M.R. \& Wurtz, R.H. (1988). Pursuit and optokinetic deficits following chemical lesions of cortical areas MT and MST. Journal of Neurophysiology, 60, 940-965.

Goldberg, M.C., Lasker, A.G., Zee, D.S., Garth, E., Tien, A., \& Landa, R.J. (2002). Deficits in the initiation of eye movements in the absence of a visual target in adolescents with high functioning autism. Neuropsychologia, 40, 2039-2049.

Heide, W., Kurzidim, K., \& Kompf, D. (1996). Deficits of smooth pursuit eye movements after frontal and parietal lesions. Brain, 119, 1951-1969.

Ilg, U.J. (1997). Slow eye movements. Progress in Neurobiology, 53, 293-329.

Ilg, U.J. \& Schumann, S. (2007). Primate area MST-1 is involved in the generation of goal-directed eye and hand movements. Journal of Neurophysiology, 97, 761-771.

Johnson, M.H. (1990). Cortical maturation and the development of visual attention in early infancy. Journal of Cognitive Neuroscience, 2, 81-95.

Kemner, C., van der Geest, J.N., Verbaten, M.N., \& van Engeland, H. (2004). In search of neurophysiological markers of pervasive developmental disorders: Smooth pursuit eye movements? Journal of Neural Transmission, 111, 1617-1626.

Levitt, H. (1971). Transformed up-down methods in psychoacoustics. Journal of the Acoustic Society of America, 49, 467-477

Lisberger, S.G. \& Pavelko, T.A. (1989). Topographic and directional organization of visual motion inputs for the initiation of horizontal and vertical smooth-pursuit eye movements in monkeys. Journal of Neurophysiology, 61, 173-185. 
Lord, C., Risi, S., Lambrecht, L., Cook, E.H., Jr., Leventhal, B.L., DiLavore, P.C., Pickles, A., \& Rutter, M. (2000). The autism diagnostic observation schedule-generic: A standard measure of social and communication deficits associated with the spectrum of autism. Journal of Autism and Developmental Disorders, 30, 205-223.

Lord, C., Rutter, M., Goode, S., Heemsbergen, J., Jordan, H., Mawhood, L., \& Schopler, E. (1989). Autism diagnostic observation schedule: A standardized observation of communicative and social behavior. Journal of Autism \& Developmental Disorders, 19, 185-212.

Lord, C., Rutter, M., \& Le Couteur, A. (1994). Autism diagnostic interview-revised: A revised version of a diagnostic interview for caregivers of individuals with possible pervasive developmental disorders. Journal of Autism \& Developmental Disorders, 24, 659-685.

Luna, B., Doll, S.K., Hegedus, S.J., Minshew, N.J., \& Sweeney, J.A. (2007). Maturation of executive function in autism. Biological Psychiatry, 61, 474-481.

Lynch, J.C. (1987). Frontal eye field lesions in monkeys disrupt visual pursuit. Experimental Brain Research, 68, 437-441.

Milne, E., Swettenham, J., Hansen, P., Campbell, R., Jeffries, H., \& Plaisted, K. (2002). High motion coherence thresholds in children with autism. Journal of Child Psychology and Psychiatry and Allied Disciplines, 43, 255-263.

Milne, E., White, S., Campbell, R., Swettenham, J., Hansen, P., \& Ramus, F. (2006). Motion and form coherence detection in autistic spectrum disorder: Relationship to motor control and 2:4 digit ratio. Journal of Autism and Developmental Disorders, 36, 225-237.

Minshew, N.J., Goldstein, G., \& Siegel, D.J. (1997). Neuropsychologic functioning in autism: Profile of a complex information processing disorder. Journal of the International Neuropsychological Society, 3, 303-316.

Minshew, N.J., Sung, K., Jones, B.L., \& Furman, J.M. (2004). Underdevelopment of the postural control system in autism. Neurology, 63, 2056-2061.

Molloy, C.A., Dietrich, K.N., \& Bhattacharya, A. (2003). Postural stability in children with autism spectrum disorder. Journal of Autism and Developmental Disorders, 33, 643-652.

Mostofsky, S.H., Goldberg, M.C., Landa, R.J., \& Denckla, M.B. (2000). Evidence for a deficit in procedural learning in children and adolescents with autism: Implications for cerebellar contribution. Journal of the International Neuropsychological Society, 6, 752-759.

Müller, R.A., Cauich, C., Rubio, M.A., Mizuno, A., \& Courchesne, E. (2004). Abnormal activity patterns in premotor cortex during sequence learning in autistic patients. Biological Psychiatry, 56, 323-332.

Newsome, W.T., Wurtz, R.H., Dursteler, M.R., \& Mikami, A. (1985). Deficits in visual motion processing following ibo- tenic acid lesions of the middle temporal visual area of the macaque monkey. Journal of Neuroscience, 5, 825-840.

Redcay, E. (2008). The superior temporal sulcus performs a common function for social and speech perception: Implications for the emergence of autism. Neuroscience and Biobehavioral Review, 32, 123-142.

Rinehart, N.J., Bradshaw, J.L., Brereton, A.V., \& Tonge, B.J. (2001). Movement preparation in high-functioning autism and Asperger disorder: A serial choice reaction time task involving motor reprogramming. Journal of Autism and Developmental Disorders, 31, 79-88.

Rivaud, S., Muri, R.M., Gaymard, B., Vermersch, A.I., \& PierrotDeseilligny, C. (1994). Eye movement disorders after frontal eye field lesions in humans. Experimental Brain Research, 102, 110-120.

Rosenhall, U., Johansson, E., \& Gillberg, C. (1988). Oculomotor findings in autistic children. Journal of Laryngology and Otology, 102, 435-439.

Scharre, J.E. \& Creedon, M.P. (1992). Assessment of visual function in autistic children. Optometry and Vision Science, 69, 433-439.

Shi, D., Friedman, H.R., \& Bruce, C.J. (1998). Deficits in smoothpursuit eye movements after muscimol inactivation within the primate's frontal eye field. Journal of Neurophysiology, 80, $458-464$.

Spencer, J., O’Brien, J., Riggs, K., Braddick, O., Atkinson, J., \& Wattam-Bell, J. (2000). Motion processing in autism: Evidence for a dorsal stream deficiency. Neuroreport, 11, 2765-2767.

Spencer, J.V. \& O’Brien, J.M. (2006). Visual form-processing deficits in autism. Perception, 35, 1047-1055.

Tager-Flusberg, H. \& Joseph, R.M. (2003). Identifying neurocognitive phenotypes in autism. Philosophical Transactions of the Royal Society London B: Biological Sciences, 358, 303-314.

Takarae, Y., Minshew, N.J., Luna, B., Krisky, C.M., \& Sweeney, J.A. (2004a). Pursuit eye movement deficits in autism. Brain, 127, 2584-2594.

Takarae, Y., Minshew, N.J., Luna, B., \& Sweeney, J.A. (2004b). Oculomotor abnormalities parallel cerebellar histopathology in autism. Journal of Neurology, Neurosurgery and Psychiatry, 75, 1359-1361.

Tsermentseli, S., O’Brien, J.M., \& Spencer, J.V. (in press). Comparison of Form and Motion Coherence Processing in Autistic Spectrum Disorders and Dyslexia. Journal of Autism and Developmental Disorders.

Williams, D.L., Goldstein, G., \& Minshew, N.J. (2006). Neuropsychologic functioning in children with autism: Further evidence for disordered complex information-processing. Child Neuropsychology, 12, 279-298.

Zilbovicius, M., Meresse, I., Chabane, N., Brunelle, F., Samson, Y., \& Boddaert, N. (2006). Autism, the superior temporal sulcus and social perception. Trends in Neuroscience, 29, 359-366. 